\title{
Reacciones adversas asociadas a vacunas contra la COVID-19
}

\section{Adverse reactions associated with vaccines against COVID-19}

Natalia Aguirre-Morales ${ }^{1 凶}$, Catalina Gómez-Henao ${ }^{2}$, Ana María Calle ${ }^{3}$, Ricardo Cardona-Villa ${ }^{4}$, Libia Susana Diez-Zuluaga ${ }^{5}$, Luis Carlos Santamaría-Salazar ${ }^{3}$, Ruth Helena Ramírez-Giraldo ${ }^{5}$, Carlos Fernando Chinchilla ${ }^{5}$, Jorge Sánchez ${ }^{5}$

${ }^{1}$ Médica, Residente de Alergología Clínica, Universidad de Antioquia. Miembro del Grupo de Alergología Clínica y Experimental (GACE), Universidad de Antioquia, Medellín, Colombia.

${ }^{2}$ Alergólogo Clínico Clínica SOMER. Miembro del Grupo de Alergología Clínica y Experimental (GACE), Universidad de Antioquia, Medellín, Colombia.

${ }^{3}$ Alergólogo Clínico, Hospital Universitario San Vicente Fundación. Miembro del Grupo de Alergología Clínica y Experimental (GACE), Universidad de Antioquia, Medellín, Colombia.

${ }^{4}$ Coordinador del Posgrado Alergología Clínica. Director Grupo de Alergología Clínica y Experimental. Miembro del Grupo de Alergología Clínica y Experimental (GACE), Universidad de Antioquia, Medellín, Colombia.

${ }^{5}$ Alergólogo Clínico IPS Universitaria. Miembro del Grupo de Alergología Clínica y Experimental (GACE), Universidad de Antioquia, Medellín, Colombia.

Fecha correspondencia:

Recibido: mayo 31 de 2021.

Revisado: agosto 13 de 2021.

Aceptado: septiembre 10 de 2021.

Forma de citar:

Aguirre-Morales N, Gómez-Henao C, Calle AM, Cardona-Villa R, DiezZuluaga LS, Santamaría-Salazar LC et al. Reacciones adversas asociadas a vacunas contra la COVID-19. Rev CES Med. 2021; 35(3): 230-243.

https://dx.doi.org/10.21615/ cesmedicina.6282

Open access

(C) Derecho de autor

Licencia creative commons Ética de publicaciones

\section{Resumen}

Con el advenimiento de la pandemia por COVID-19 se generó la necesidad de diseñar estrategias que ayudaran a mitigar la morbimortalidad causada por el virus y una de las más prometedoras es la vacunación masiva. Sin embargo, la vacunación puede asociarse a reacciones adversas, entre ellas, reacciones de hipersensibilidad a los componentes de las diferentes vacunas, por lo que es fundamental conocer dichos $c$ omponentes y la población que requiere una valoración previa por Alergología. Todo paciente que lo requiera se debe remitir oportunamente con el fin de reconocer el componente implicado en la reacción por medio de pruebas adecuadas y ofrecer una conducta que permita continuar un esquema de vacunación que sea seguro. Este artículo busca recopilar los datos de reacciones adversas, con énfasis en reacciones de hipersensibilidad, que se han presentado en ensayos clínicos con la aplicación de las vacunas contra el SARS-CoV-2 para ser 
Revisión por pares

Gestión por Open Journal

System

DOI:

10.21615/cesmedicina.6282

ISSNe 2215-9177

ISSN 0120-8705

Publica con nosotros aplicadas en Colombia. Adicionalmente, se realiza una propuesta de abordaje de los pacientes con antecedentes de reacciones de hipersensibilidad con respecto a la conducta que se debe tomar para su vacunación.

Palabras clave: vacuna; COVID-19; hipersensibilidad; alergia; reacciones adversas.

\section{Abstract}

With the occurrence of the COVID-19 pandemic, the need to design strategies to help mitigate the morbimortality caused by the virus arose and one of the most promising is mass vaccination. However, vaccination may be associated with adverse reactions, including hypersensitivity reactions to the components of the different vaccines, so it is essential to know these components and the population that requires prior assessment by Allergology. Any patient who requires it should be referred in a timely manner, in order to recognize the component involved in the reaction by means of appropriate tests and to offer a course of action that allows continuing a safe vaccination schedule. This article seeks to compile data on adverse reactions, with emphasis on hypersensitivity reactions, which have occurred in clinical trials with the application of vaccines against SARS-CoV-2 to be applied in Colombia. Additionally, a proposal is made to approach patients with a history of hypersensitivity reactions with respect to the conduct that should be taken for their vaccination.

Keywords: vaccines; COVID-19; hypersensitivity; allergy; adverse reactions.

\section{Introducción}

El 31 de diciembre de 2019 se notificó en Wuhan, China, un brote causado por un virus que luego se conocería como coronavirus del síndrome respiratorio agudo grave tipo 2, responsable de la enfermedad conocida como COVID-19 (Coronavirus disease) ${ }^{(1-3)}$. La alta tasa de transmisión entre humanos llevó a que el 11 de marzo de 2020, 113 países del mundo tuvieran casos confirmados de dicha infección y la Organización Mundial de la Salud la declarara pandemia ${ }^{(4-5)}$. Al 3 de marzo de 2021 se han reportado un total de 115 millones de casos y 2,56 millones de muertes para una tasa de mortalidad de $2,22 \%{ }^{(6)}$. 
Septiembre - diciembre de 2021

Desde principios de 2020 se empezó el desarrollo de vacunas capaces de mitigar el impacto de esta enfermedad. Las vacunas convencionales contienen proteínas virales o formas inactivas del propio virus que estimulan la generación de memoria inmunológica. Sin embargo, algunas vacunas contra la COVID-19, cuya eficacia se notificó en ensayos clínicos de fase III ${ }^{7,8)}$, utilizan una cadena de ARNm (ácido ribonucleico mensajero) que codifica la proteína espiga del SARS-CoV-2, dentro de una capa lipídica. Una vez el ARNm ingresa a las células humanas se inicia la transcripción de la proteína codificada en este material genético. Esto actúa como el antígeno que desencadena la respuesta inmune. En diciembre de 2020 una vacuna fabricada por la casa farmacéutica Pfizer y la empresa alemana de biotecnología BioNTech, recibió aprobación para uso de emergencia por la FDA ${ }^{(9)}$. Las vacunas fabricadas por Pfizer y BioNTech y por la compañía farmacéutica estadounidense Moderna utilizan ARNm que codifica la proteína espiga ${ }^{(9)}$.

En noviembre de 2020 se publicó el ensayo clínico fase III de la vacuna fabricada por la empresa farmacéutica AstraZeneca y la Universidad de Oxford. En el caso de esta vacuna se utiliza un vector viral que contiene material genético adicional que codifica la proteína espiga del SARS-CoV-2; este es el mismo mecanismo de acción que utiliza la vacuna de la empresa Janssen ${ }^{(9)}$. Por otra parte, la vacuna CoronaVac, desarrollada por el laboratorio Sinovac, utiliza el virus inactivado con un adyuvante a base de aluminio para estimular la inmunidad protectora ${ }^{(10)}$. Muchas otras vacunas se vienen desarrollando con diferente grado de comprobación de su eficacia, cada una con tecnologías particulares, lo que abre la posibilidad de nuevas herramientas terapéuticas.

De acuerdo al plan de vacunación 2021 en Colombia se tiene programada la aplicación de vacunas de los laboratorios Pfizer, AstraZeneca, Johnson \& Johnson, Moderna y Sinovac. En la tabla 1 , se resumen las principales características de estas cinco vacunas, incluyendo su mecanismo de acción, esquema de dosificación y principales excipientes. Sin embargo, con la posibilidad de vacunación por parte del sector privado es posible que la oferta se amplíe a futuro $^{(11)}$. 
Tabla 1. Resumen de vacunas disponibles en el territorio colombiano.

\begin{tabular}{|c|c|c|c|}
\hline $\begin{array}{c}\text { Desarrollador (nombre } \\
\text { de la vacuna) }\end{array}$ & Mecanismo de acción & $\begin{array}{l}\text { Esquema de } \\
\text { dosificación }\end{array}$ & Principales excipientes \\
\hline $\begin{array}{l}\text { BioNTech-Pfizer } \\
\text { (BNT162b2) }\end{array}$ & $\begin{array}{l}\text { ARNm que codifica la } \\
\text { proteína espiga del } \\
\text { SARSCoV2 }\end{array}$ & $\begin{array}{l}\text { Dos dosis } \\
\text { (día } 0 \text { y } 21 \text { ) }\end{array}$ & $\begin{array}{l}\text { PEG, 1,2-diestearoil-sn-glicero-3- } \\
\text { fosfocolina, colesterol, cloruro de } \\
\text { potasio, fosfato de potasio } \\
\text { monobásico, cloruro de sodio, } \\
\text { fosfato de sodio dibásico dihidrato y } \\
\text { sacarosa. }\end{array}$ \\
\hline $\begin{array}{l}\text { AstraZeneca y } \\
\text { Universidad de Oxford } \\
\text { (ChAdOx1 nCoV-19) }\end{array}$ & $\begin{array}{l}\text { Vector (adenovirus) no } \\
\text { replicativo que } \\
\text { expresa la proteína } \\
\text { espiga del SARSCoV2 }\end{array}$ & $\begin{array}{l}\text { Dos dosis } \\
\text { (días } 0 \text { y } \\
70-84 \text { ) }\end{array}$ & $\begin{array}{l}\text { Histidina, sacarosa, cloruro de sodio, } \\
\text { cloruro de magnesio, polisorbato } 80, \\
\text { edetato disódico y etanol }\end{array}$ \\
\hline $\begin{array}{l}\text { Johnson \& Johnson } \\
\text { (Janssen/Ad26.COV2.S) }\end{array}$ & $\begin{array}{l}\text { Vector (adenovirus) no } \\
\text { replicativo que } \\
\text { expresa la proteína } \\
\text { espiga del SARSCoV2 }\end{array}$ & Una dosis & $\begin{array}{l}\text { Cloruro de sodio, ácido cítrico } \\
\text { monohidrato, polisorbato } 80,2 \\
\text { hidroxipropil-B-ciclodextrina, etanol } \\
\text { e hidróxido de sodio }\end{array}$ \\
\hline Moderna (mRNA-1273) & $\begin{array}{l}\text { ARNm que codifica la } \\
\text { proteína espiga del } \\
\text { SARSCoV2 }\end{array}$ & $\begin{array}{l}\text { Dos dosis } \\
\text { (días } 0 \text { y } 28 \text { ) }\end{array}$ & $\begin{array}{l}\text { PEG, Lípidos, trometamina, } \\
\text { clorhidrato de trometamina, ácido } \\
\text { acético, acetato de sodio y sacarosa }\end{array}$ \\
\hline Sinovac (CoronaVac) & Virus inactivo & $\begin{array}{l}\text { Dos dosis } \\
\text { (días } 0 \text { y } 56 \text { ) }\end{array}$ & $\begin{array}{l}\text { Hidróxido de aluminio, } \\
\text { hidrogenofosfato de disodio, } \\
\text { dihidrogenofosfato de sodio, cloruro } \\
\text { de sodio }\end{array}$ \\
\hline
\end{tabular}

Adaptado de ref (12) PEG: Polietilenglicol.

Según la OMS, las reacciones adversas a medicamentos, incluyendo vacunas, se definen como el conjunto de efectos adversos no deseados ocasionados por fármacos que ocurre tras la administración de una dosis medicamentosa normalmente utilizada en la especie humana para profilaxis, diagnóstico o tratamiento de la enfermedad o para modificación de una función biológica. Entre estas, se incluyen las reacciones de hipersensibilidad mediadas por mecanismos inmunológicos conocidos o no. Las reacciones de hipersensibilidad son aquellas cuyo efecto no es explicable por la acción farmacológica que induce una respuesta anormal mediada por un mecanismo inmunológico. Se caracteriza por su especificidad, mediada por anticuerpos o linfocitos y que se reproduce con lo exposición al fármaco ${ }^{(13)}$. 
Septiembre - diciembre de 2021

A continuación, se detallan las reacciones adversas identificadas en estudios de fase 3 de las vacunas disponibles en el territorio nacional, con énfasis en reacciones de hipersensibilidad.

\section{Reacciones adversas reportadas en estudios fase 3}

\section{BioNTech - Pfizer (BNT162b2)}

El estudio de fase III de la vacuna de Pfizer fue publicado en diciembre de $2020{ }^{(8)}$ y reporta los resultados de la aleatorización 1:1 de 43448 personas para recibir placebo o la vacuna. En general, se reportaron más eventos adversos en el grupo de pacientes que recibieron la vacuna comparado con placebo (26,7 \% vs $12,2 \%)$. Entre los pacientes que recibieron la vacuna, el dolor leve a moderado en el sitio de inyección fue la reacción local más reportada y menos del $1 \%$ de los participantes informaron dolor intenso. Los eventos adversos graves se presentaron en el $1,1 \%$ de los pacientes que recibieron la vacuna y $0,6 \%$ de los pacientes que recibieron placebo, estos eventos adversos no se detallan en los resultados del estudio de fase 3.

En el reporte de resultados no se describe la incidencia de reacciones de hipersensibilidad incluyendo reacciones anafilácticas. Se reportaron dos muertes en el grupo de pacientes que recibió la vacuna, por causas no relacionadas con la aplicación del biológico y cuatro en el grupo placebo, de las cuales tampoco de detallan las causas ni en el estudio publicado ni en el material suplementario.

\section{AstraZeneca y Universidad de Oxford (ChAdOx1 nCoV-19)}

Con base en el estudio de fase II/III publicado en Lancet en diciembre de $2020^{(14)}$ en el cual se aleatorizaron 560 participantes, se encontró que 43 (88\%) participantes en el grupo de 18 a 55 años, 22 (73\%) en el grupo de 56 a 69 años y 30 (61 \%) en el grupo de 70 años o más presentaron al menos un síntoma local después de la vacunación con dosis estándar. Las reacciones adversas sistémicas más frecuentemente reportadas fueron: fatiga, cefalea, fiebre y mialgias. Se informó al menos un síntoma sistémico después de la primera vacunación con la dosis estándar en 42 (86 \%) participantes en el grupo de 18 a 55 años, 23 (77\%) en el grupo de 56 a 69 años grupo y 32 (65 \%) en el grupo de 70 años o más. No se reportó ningún evento compatible con una reacción de hipersensibilidad entre los pacientes de este estudio.

\section{Johnson \& Johnson (Janssen/Ad26.COV2.S)}

El ensayo clínico fase III ENSEMBLE (aún en curso) busca evaluar la eficacia y la seguridad de la vacuna diseñada por el laboratorio Johnson \& Johnson. Para ello se inició el reclutamiento en septiembre de 2020 y la meta es reclutar 40000 participantes $^{(15)}$. En febrero de 2021 se hizo 
público un documento informativo por parte del laboratorio, reportando los datos del ensayo clínico hasta el momento. En este reportan que las reacciones de hipersensibilidad inmediata después de la vacunación fueron raras, no graves y no había casos de anafilaxia. Las reacciones vasovagales como el síncope, fueron raras $(0,1 \%)$ y se distribuyeron uniformemente entre el grupo que recibió la vacuna y el placebo.

Los eventos adversos que ocurrieron dentro de los 30 minutos posteriores a la vacunación fueron poco frecuentes; $0,5 \%$ en el grupo que recibió la vacuna vs $0,3 \%$ en el grupo placebo. Hasta el momento, en cuanto a reacciones inmediatas, se han descrito 24 casos de brote maculopapular posterior a la aplicación de la vacuna y 16 con placebo, así como ocho casos de urticaria y tres en el grupo placebo ${ }^{(16)}$.

\section{Moderna (mRNA-1273)}

El ensayo fase III reclutó a 30420 voluntarios que fueron asignados al azar 1:1 para recibir la vacuna o placebo (15 210 participantes en cada grupo). Se informaron reacciones de hipersensibilidad en el $1,5 \%$ y el $1,1 \%$ en los grupos de vacuna y placebo, respectivamente. En el material suplementario se detallan dichas reacciones de la siguiente manera: un paciente con anafilaxia en el grupo placebo y uno en el grupo de pacientes que recibió la vacuna $(<0,1 \%$ en ambos casos), tres reportes de angioedema con el placebo y una con la vacuna; brote cutáneo no especificado en 34 pacientes del grupo placebo $(0,2 \%)$ y 45 pacientes del grupo que recibió la vacuna $(0,3 \%)$. Adicionalmente, reportan que 23 pacientes en el grupo placebo $(0,2 \%)$ presentaron urticaria tardía vs 27 pacientes $(0,2 \%)$ en el grupo que recibió la vacuna ${ }^{(7)}$.

\section{CoronaVac (Sinovac)}

Al momento, no hay una publicación oficial de los resultados de estudios fase III de la vacuna CoronaVac del laboratorio chino Sinovac. El primero de febrero de 2021 se publicaron en Lancet los resultados de los estudios fase I y II ${ }^{(16)}$. En la fase I, los participantes se asignaron al azar para recibir la vacuna o placebo. En la fase II, los participantes se asignaron al azar a CoronaVac en dosis baja, en dosis alta o placebo. Se incluyeron un total de 743 participantes que recibieron por lo menos una dosis.

Solo se reportó un caso de hipersensibilidad manifestada como urticaria 48 horas después de la primera dosis en el estudio de fase II, tras recibir la dosis alta de la vacuna. El participante recibió clorfeniramina y dexametasona y se recuperó en tres días y no se observó una reacción similar después de la segunda dosis de vacuna. No se observaron eventos adversos graves relacionados con la vacuna dentro de los 28 días posteriores a la vacunación. 


\section{Propuesta de abordaje de pacientes con antecedentes alergias}

En enero de 2021, la Organización Mundial de Alergias publicó el concepto del comité de anafilaxia con respecto al posible origen alérgico de las reacciones asociadas a la aplicación de la vacuna contra la COVID-19 ${ }^{(10)}$. La figura 1 resume la propuesta de abordaje de pacientes con antecedentes de reacciones o enfermedades de origen alérgico con respecto a la vacunación. Se define una reacción alérgica grave como una reacción que requirió hospitalización o prolongación de la hospitalización, fue incapacitante o limitó las actividades de la vida diaria o una reacción con consecuencias potencialmente mortales.

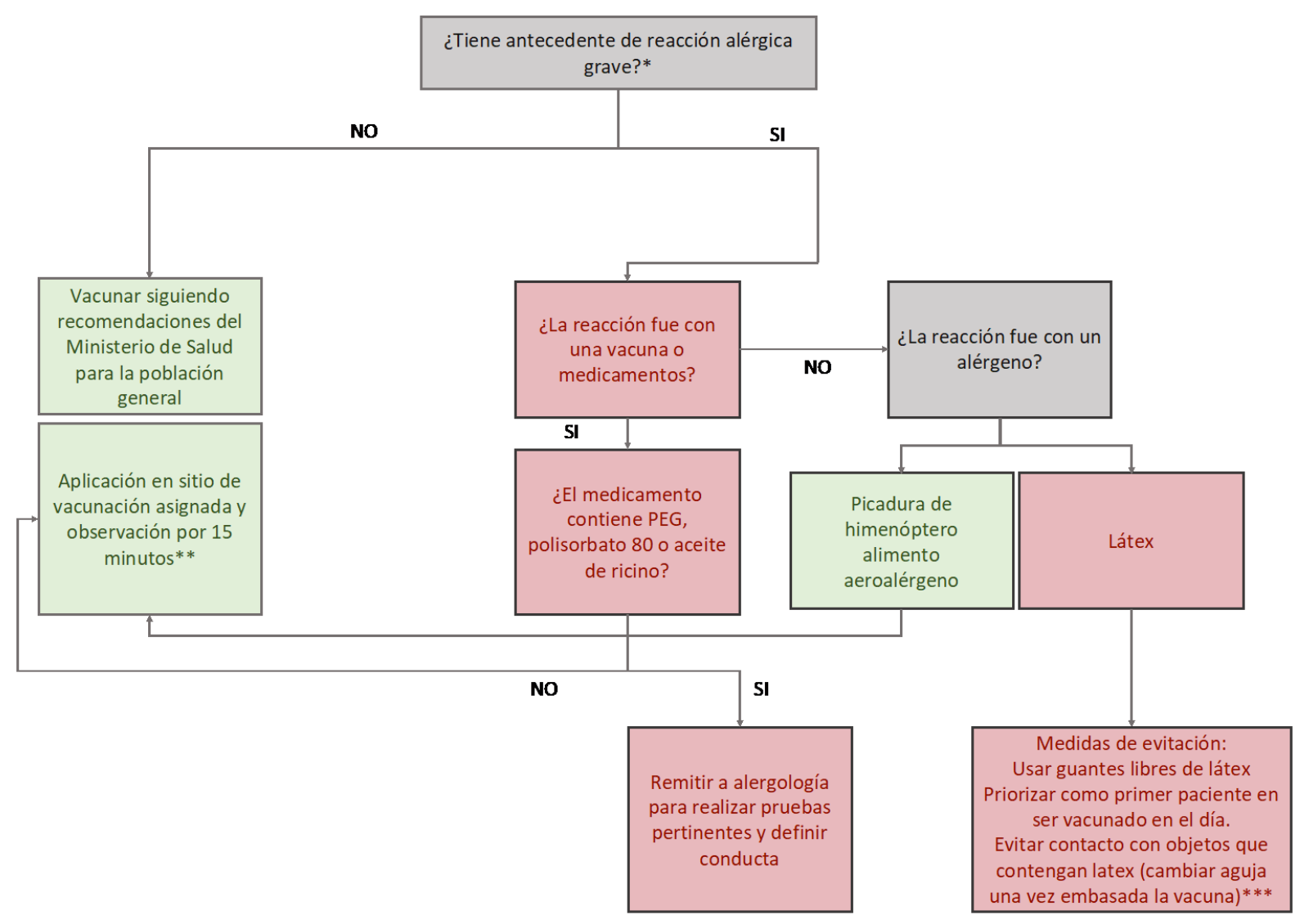

Figura 1. Propuesta de conducta a seguir con base en los antecedentes de cada paciente.

* Reacción alérgica grave: reacción que requirió hospitalización o prolongación de la hospitalización, fue incapacitante o limitó las actividades de la vida diaria o de autocuidado. Reacción con consecuencias potencialmente mortales requiriendo intervención urgente ${ }^{(20)}{ }^{* *(21)}{ }^{* * *(22)}$

PEG: Polietilenglicol. 
En todos los casos, independientemente de los antecedentes de los pacientes, la aplicación de la vacuna debe ser en un centro que cuente con personal entrenado en el reconocimiento oportuno y tratamiento de reacciones alérgicas, incluyendo reacciones potencialmente mortales como la anafilaxia. Esta es una recomendación preventiva y es posible que a futuro pase a ser innecesaria en el contexto de una vacunación masiva y con base en la evidencia que se haga disponible. En caso de presentarse, la anafilaxia postvacunal debe tratarse con adrenalina intramuscular y medidas generales dependiendo de las manifestaciones de cada paciente ${ }^{(18,19)}$.

\section{Pruebas alergológicas en pacientes con reacciones de hipersensibilidad postvacunales}

Ante la sospecha de una reacción de hipersensibilidad inmediata con la aplicación de la vacuna contra la COVID-19 (primeras seis horas luego de su aplicación) ${ }^{(2)}$, es importante seguir un esquema secuencial que ayude a identificar la sustancia implicada en la reacción. Si se trata de una anafilaxia, se debe solicitar medición de triptasa sérica en las primeras dos horas de la reacción y posteriormente pasadas 24 horas de esta ${ }^{(23)}$.

Es importante diferenciar las reacciones vasovagales de las reacciones anafilácticas (tabla 2$)^{(18)}$. Se debe revisar cada uno de los compuestos de la vacuna implicada para realizar los estudios alergológicos pertinentes. Dos de los compuestos que se deben estudiar en el contexto de alergia a la vacuna contra la COVID-19 son el polietilenglicol (PEG) y el polisorbato-80 (24). Los polietilenglicoles y los polisorbatos son sustancias relacionadas estructuralmente dado su grupo poliéter. Estos compuestos tienen una gran variedad de usos en la industria de medicamentos y se han visto implicados en reacciones de hipersensibilidad inmediatas. Entre sus múltiples usos, los polietilenglicoles con peso molecular entre 400 y 6000 se usan como excipientes en muchas formulaciones líquidas y sólidas de medicamentos, incluyendo la preparación intestinal para colonoscopias ${ }^{(26,27,28)}$. 
Tabla 2. Características de las reacciones vasovagales y reacciones de anafilaxia.

\begin{tabular}{cll}
\hline Signos y síntomas & \multicolumn{1}{c}{ Reacción vasovagal } & \multicolumn{1}{c}{ Anafilaxia } \\
\hline $\begin{array}{c}\text { Intervalo (después } \\
\text { de la inyección) }\end{array}$ & $\begin{array}{l}\text { A veces antes, generalmente } \\
\text { después de unos segundos a } \\
\text { minutos después de la inyección. }\end{array}$ & $\begin{array}{l}\text { Dentro de los } 30 \text { minutos posteriores a la } \\
\text { inyección; las reacciones más graves } \\
\text { comienzan dentro de los primeros } 15 \text { minutos }\end{array}$ \\
\hline Consciencia & $\begin{array}{l}\text { Lipotimia, mareos, o pérdida de la } \\
\text { consciencia en algunos casos. }\end{array}$ & $\begin{array}{l}\text { Alteración del sensorio, que puede progresar } \\
\text { hasta la pérdida de la consciencia en casos } \\
\text { graves }\end{array}$ \\
\hline Respiración & $\begin{array}{l}\text { Lenta, con algunos segundos de } \\
\text { apnea, en algunos casos }\end{array}$ & $\begin{array}{l}\text { Dificultad respiratoria: tos, estornudos, } \\
\text { sibilancias, estridor }\end{array}$ \\
\hline Pulso & Lento y débil, pero regular & Rápido, débil e irregular \\
\hline Piel & Diaforesis, piel húmeda, palidez & $\begin{array}{l}\text { Piel cálida, que progresa a húmeda y palidez. } \\
\text { Prurito y urticaria (>90 \% de los casos) } \\
\text { Angioedema de cara y lengua }\end{array}$ \\
\hline Sistema & Hipotensión transitoria & $\begin{array}{l}\text { Hipotensión (PAS <90 mm Hg), que puede } \\
\text { progresar a colapso cardiovascular }\end{array}$ \\
\hline gastrointestinal & Náuseas, vómitos & $\begin{array}{l}\text { Náuseas, vómitos, dolores abdominales, } \\
\text { diarrea }\end{array}$ \\
\hline
\end{tabular}

Adaptado de ref (23).

En la tabla 3 se detallan los estudios propuestos para abordar la sospecha de alergia al polietilenglicol. Para esto, se sugiere el uso de acetato de metilprednisolona. Como control negativo se sugiere el uso de succinato de sodio de metilprednisolona que no contiene polietilenglicol y utilizando las mismas concentraciones que se utilizan para las pruebas con acetato de metilprednisolona ${ }^{(25)}$.

Este abordaje es el recomendado por diferentes artículos de posición ${ }^{(25,28)}$ con respecto al tema, pero aún no hay certeza de que estas sustancias realmente son las implicadas. Al momento tampoco contamos con estudios de rendimiento diagnóstico de estas pruebas. 
Tabla 3. Protocolo para pruebas cutáneas con acetato de metilprednisolona (polietilenglicol 3350).

\begin{tabular}{|c|c|c|c|}
\hline & Paso 1 & Paso 2 & Paso 3 \\
\hline Prueba intraepidérmica (se puede realizar también con PEG sin diluir) & $40 \mathrm{mg} / \mathrm{mL}$ & & \\
\hline Prueba intradérmica & & $0,4 \mathrm{mg} / \mathrm{mL}$ & \\
\hline Prueba intradérmica & & & $4 \mathrm{mg} / \mathrm{mL}$ \\
\hline
\end{tabular}

Para estudiar la posibilidad de que la reacción sea debida al polisorbato 80 se sugiere usar gotas oftálmicas lubricantes que contengan dicho componente (Optive Advanced ${ }^{\circledR}$ ) o acetónido de triamcinolona y realizar las pruebas (tabla 4) ${ }^{(23)}$. Es importante recordar que tanto el polietilenglicol como el polisorbato-80 son compuestos presentes en una gran cantidad de medicamentos, incluyendo algunos que se usan con frecuencia en el ámbito de urgencias o para contrarrestar reacciones de hipersensibilidad ${ }^{(26)}$. Ante la confirmación de reacciones cutáneas positivas ya sea en el estudio de polisorbato-80 o PEG se sugiere evitar la administración de vacunas que contengan alguno de estos dos componentes.

Tabla 4. Concentraciones no irritativas para realizar pruebas cutáneas con polisorbato 80 .

\begin{tabular}{ccc}
\hline Tipo de prueba & $\begin{array}{c}\text { Concentración de gotas oftálmicas } \\
\text { con polisorbato } 80 \text { al 5 \% }\end{array}$ & $\begin{array}{c}\text { Concentración de acetónido } \\
\text { de triamcinolona }\end{array}$ \\
\hline Prueba intraepidérmica & $1: 1$ & $40 \mathrm{mg} / \mathrm{mL}$ \\
\hline Prueba intradérmica & $1: 10$ & $0,4 \mathrm{mg} / \mathrm{mL}$ \\
\hline Prueba intradérmica & & $4 \mathrm{mg} / \mathrm{mL}$ \\
\hline Prueba intradérmica & & $40 \mathrm{mg} / \mathrm{mL}$ \\
\hline
\end{tabular}

\section{Conclusión}

Ante el inicio de la vacunación masiva contra la COVID-19 se generan dudas sobre la seguridad de las vacunas en pacientes con antecedentes de alergias. La aplicación de las vacunas no debe ser obstaculizada en pacientes que no tengan contraindicaciones, siempre teniendo en cuenta seguir un abordaje sistemático que permita identificar a aquellos que requieran valoración previa. 
En esta revisión se hacen recomendaciones para la realización de pruebas cutáneas para dos sustancias en las vacunas que son sospechosas en causar reacciones alérgicas; sin embargo, aún no hay estudios que confirmen el riesgo de que estas sean las sustancias verdaderamente implicadas. En el estudio de Greenhawt et al., se enfatiza en la necesidad de continuar investigando la seguridad y el rendimiento de las pruebas utilizadas para el diagnóstico de reacciones de hipersensibilidad con vacunas contra SarsCov2 y sus excipientes. Por ende, es de esperarse que la evidencia de este tema esté en un constante proceso de actualización ${ }^{(29)}$.

\section{Bibliografía}

1. Whitney CG, Zhou F, Singleton J, Schuchat A, Centers for Disease Control and Prevention (CDC). Benefits from immunization during the vaccines for children program era - United States, 1994-2013. MMWR Morb Mortal Wkly Rep. 2014;63(16):352-5.

2. Nilsson L, Brockow K, Alm J, Cardona V, Caubet J-C, Gomes E, et al. Vaccination and allergy: EAACl position paper, practical aspects. Pediatr Allergy Immunol. 2017;28(7):628-40.

3. Dreskin SC, Halsey NA, Kelso JM, Wood RA, Hummell DS, Edwards KM, et al. International Consensus (ICON): allergic reactions to vaccines. World Allergy Organ J. 2016;9(1):32.

4. Coronaviridae Study Group of the International Committee on Taxonomy of Viruses. The species Severe acute respiratory syndrome-related coronavirus: classifying 2019nCoV and naming it SARS-CoV-2. Nat Microbiol. 2020;5(4):536-44.

5. Martellucci CA, Flacco ME, Cappadona R, Bravi F, Mantovani L, Manzoli L. SARS-CoV-2 pandemic: An overview [Internet]. Vol. 77, Advances in Biological Regulation. 2020. p. 100736. Available from: http://dx.doi.org/10.1016/j.jbior.2020.100736

6. Our World in Data. Cumulative confirmes COVID-19 deaths. Global Change Data Lab. Website [Internet]. [cited 2021]. Available from: https://ourworldindata.org/grapher/cumulative-covid-deathsregion?tab=chart\&stackMode=absolute\&time=2020-01-11..latest $\&$ region=World

7. Baden LR, El Sahly HM, Essink B, Kotloff K, Frey S, Novak R, et al. Efficacy and Safety of the mRNA-1273 SARS-CoV-2 Vaccine. N Engl J Med. 2021;384(5):403-16. 
8. Polack FP, Thomas SJ, Kitchin N, Absalon J, Gurtman A, Lockhart S, et al. Safety and Efficacy of the BNT162b2 mRNA Covid-19 Vaccine. N Engl J Med. 2020;383(27):260315.

9. Ball P. The lightning-fast quest for COVID vaccines - and what it means for other diseases [Internet]. Vol. 589, Nature. 2021. p. 16-8. Available from: http://dx.doi.org/10.1038/d41586-020-03626-1

10. Turner PJ, Ansotegui IJ, Campbell DE, Cardona V, Ebisawa M, El-Gamal Y, et al. COVID19 vaccine-associated anaphylaxis: A statement of the World Allergy Organization Anaphylaxis Committee. World Allergy Organ J. 2021;14(2):100517.

11. Ministerio de Salud y Protección Social. Vacunación contra COVID-19. Website [Internet]. [cited 2021]. Available from: https://www.minsalud.gov.co/salud/publica/Vacunacion/Paginas/Vacunacion-covid19.aspx

12. Castells MC, Phillips EJ. Maintaining Safety with SARS-CoV-2 Vaccines. Reply. N Engl J Med. 2021;384(10): e37.

13. WHO Drug and Therapeutics Committee Training Course. Assessing and Managing Medicine Safety [Internet]. Website [Internet]. [cited 2021]. Available from: https://www.who.int/medicines/technical briefing/tbs/04-PG Dug-Safety final08.pdf?ua=1

14. Ramasamy MN, Minassian AM, Ewer KJ, Flaxman AL, Folegatti PM, Owens DR, et al. Safety and immunogenicity of ChAdOx1 nCoV-19 vaccine administered in a prime-boost regimen in young and old adults (COV002): a single-blind, randomised, controlled, phase 2/3 trial. Lancet. 2021;396(10267):1979-93.

15. U.S. National Library of Medicine. A Study of Ad26.COV2.S for the Prevention of SARSCoV-2-Mediated COVID-19 in Adult Participants (ENSEMBLE). Website [Internet]. [cited 2021]. Available from: https://clinicaltrials.gov/ct2/show/NCT04505722

16. Browne SK, Beeler JA, Roberts JN. Summary of the Vaccines and Related Biological Products Advisory Committee meeting held to consider evaluation of vaccine candidates for the prevention of respiratory syncytial virus disease in RSV-naïve infants [Internet]. Vol. 38, Vaccine. 2020. p. 101-6. Available from: http://dx.doi.org/10.1016/j.vaccine.2019.10.048 
17. Xia S, Zhang $Y$, Wang $Y$, Wang $H$, Yang $Y$, Gao GF, et al. Safety and immunogenicity of an inactivated SARS-CoV-2 vaccine, BBIBP-CorV: a randomised, double-blind, placebocontrolled, phase 1/2 trial. Lancet Infect Dis. 2021;21(1):39-51.

18. Cardona V, Ansotegui IJ, Ebisawa M, El-Gamal Y, Fernandez Rivas M, Fineman S, et al. World allergy organization anaphylaxis guidance 2020. World Allergy Organ J. 2020;13(10):100472.

19. Cardona V, Álvarez-Perea A, Ansotegui-Zubeldia IJ, Arias-Cruz A, Ivancevich JC, González-Díaz SN, et al. [Clinical Practice Guide for Anaphylaxis in Latin America (Galaxia-Latam)]. Rev Alerg Mex. 2019;66 Suppl 2:1-39.

20. U.S. Department of Health and Human Services. Common Terminology Criteria for Adverse Events (CTCAE). Website [Internet]. [cited 2021]. Available from: https://www.eortc.be/services/doc/ctc/CTCAE 4.03 2010-0614 QuickReference 5x7.pdf

21. Ministerio de Salud y Protección Social. Lineamientos técnicos y operativos para la vacunaci'n contra el COVID-19. [Internet]. [cited 2021]. Available from: https://www.minsalud.gov.co/sites/rid/Lists/BibliotecaDigital/RIDE/VS/lineamientostecnicos-operativos-covid19-anexos.pdf

22. Nucera E, Aruanno A, Rizzi A, Centrone M. Latex Allergy: Current Status and Future Perspectives. J Asthma Allergy. 2020;13:385-98.

23. Jimenez-Rodriguez TW, Garcia-Neuer M, Alenazy LA, Castells M. Anaphylaxis in the 21st century: phenotypes, endotypes, and biomarkers. J Asthma Allergy. 2018;11:121-42.

24. Garvey LH, Nasser S. Anaphylaxis to the first COVID-19 vaccine: is polyethylene glycol (PEG) the culprit? Br J Anaesth. 2021;126(3):e106-8.

25. Banerji A, Wickner PG, Saff R, Stone CA Jr, Robinson LB, Long AA, et al. mRNA Vaccines to Prevent COVID-19 Disease and Reported Allergic Reactions: Current Evidence and Suggested Approach. J Allergy Clin Immunol Pract. 2021;9(4):1423-37.

26. Stone CA Jr, Liu Y, Relling MV, Krantz MS, Pratt AL, Abreo A, et al. Immediate Hypersensitivity to Polyethylene Glycols and Polysorbates: More Common Than We Have Recognized. J Allergy Clin Immunol Pract. 2019;7(5):1533-40.e8. 
27. Sokolowska M, Eiwegger T, Ollert M, Torres MJ, Barber D, Del Giacco S, et al. EAACI statement on the diagnosis, management and prevention of severe allergic reactions to COVID-19 vaccines. Allergy [Internet]. 2021; Available from: http://dx.doi.org/10.1111/all.14739

28. Sellaturay P, Nasser S, Ewan P. Polyethylene Glycol-Induced Systemic Allergic Reactions (Anaphylaxis). J Allergy Clin Immunol Pract. 2021;9(2):670-5.

29. Greenhawt M, Abrams EM, Shaker M, Chu DK, Khan D, Akin C, et al. The Risk of Allergic Reaction to SARS-CoV-2 Vaccines and Recommended Evaluation and Management: A Systematic Review, Meta-Analysis, GRADE Assessment, and International Consensus Approach. The Journal of Allergy and Clinical Immunology: In Practice [Internet]. 2021; Available from: http://dx.doi.org/10.1016/j.jaip.2021.06.006 